\title{
NOUVELLE
}

\section{Les plasmocytes régulateurs naturels : identification et rôle en physiopathologie}

Quentin Simon, Simon Fillatreau
Institut Necker-enfants-malades (INEM), 14, rue Maria Héléna Vieira da Silva, 75014 Paris, France. quentin.simon@inserm.fr, simon.fillatreau@inserm.fr
> Les plasmocytes représentent une population de lymphocytes $B$ activés à un stade de différenciation terminale au cours duquel ils produisent des anticorps. Ces anticorps sont un fondement essentiel de la défense de l'organisme contre les pathogènes. Les plasmocytes peuvent également produire des cytokines anti-inflammatoires, telles que les interleukines 10 (IL$10)$ et 35 (IL-35), et agir comme modulateurs de l'inflammation, comme cela est observé dans des modèles de maladies auto-immunes, infectieuses, et de cancer $(\rightarrow)$ Voir la Synthèse de H. Kaplon et M.C. DieuNosjean, $m / s$ $n^{\circ} 1$, janvier 2018, page 72 et la Nouvelle de J.D. Bouaziz et al., $\mathrm{m} / \mathrm{s} \mathrm{n}^{\circ} \mathrm{8}-9$, août-septembre 2014, page 721 [1-3] $(\rightarrow)$.

De nombreuses inconnues persistent quant à l'identité de ces plasmocytes dits « régulateurs ». Sont-ils présents naturellement dans l'organisme? Quelle est leur origine cellulaire ? Existe-t-il un biomarqueur exclusif permettant de les identifier? Des éléments de réponse à ces questions majeures sont présentés dans cette nouvelle.

L'activation des cellules immunitaires et leur différenciation sont fortement influencées par le type de stimulus à l'origine de leur réponse. Une infection par la bactérie Salmonella Typhimurium conduit à une induction rapide de plasmocytes producteurs d'IL-10 ; ceci en fait un modèle physiopathologique favorable pour caractériser ces cellules régulatrices qui pourraient avoir un intérêt thérapeutique. Avant d'envisager la manipulation de telles cellules dans un contexte clinique, il est cependant nécessaire de caractériser plus précisément leurs propriétés.

Dans les jours suivant une infection par S. Typhimurium, la majorité des cellules produisant de I'IL-10 sont des plasmocytes qui présentent à leur surface le récepteur LAG-3 (lymphocyte activation gene-3) (Figure 1) [4]. LAG-3 est une molécule inhibitrice exprimée par différents types cellulaires dont les lymphocytes $T$ [5]. Des essais cliniques sont en cours pour tester si le blocage de LAG-3 améliore le contrôle de la maladie chez des patients souffrant de cancer, soulignant ainsi la pertinence physiopathologique de cette molécule et de ses ligands [5]. Les plasmocytes producteurs d'IL-10 expriment également PD-Ll et PD-L2 (progammed death ligand 1, 2), qui interagissent avec PD-1 (programmed cell death-1) [6]. L'inhibition de l'interaction entre PD-1 et PD-L1 ou PD-L2 par des anticorps anti-PD-1 est depuis peu utilisée avec succès dans le traitement de certains cancers tels que $(\rightarrow)$ Voir l'Éditorial de S. Champiat et J.C. Soria, $m / s$ $n^{\circ}$ 6-7, juin-juillet 2017, page 563 le mélanome dont elle a révolutionné en partie le pronostic [7] $(\rightarrow)$.

Les plasmocytes régulateurs peuvent donc utiliser plusieurs mécanismes moléculaires pour moduler la réponse immunitaire.

Le récepteur LAG-3 n'est pas indispensable à la production d'IL-10 par les plasmocytes après une infection bactérienne chez des souris puisque cette réponse est aussi observée chez les souris dont le gène Lag3 a été invalidé. De plus, l'expression de LAG-3 a aussi été observée sur environ $30 \%$ des plasmocytes de la rate de souris naïves (non infectées) ; ces derniers n'expriment cependant pas spontanément l'IL-10. C'est donc l'infection qui déclenche la production d'IL-10 par les plasmocytes $L A G-3^{+}$préexistants chez les souris naïves. L'infection induit une augmentation importante du nombre de plasmocytes totaux, mais les plasmocytes exprimant LAG-3 ne prolifèrent pas lors de cette infection. Le récepteur LAG-3 permet ainsi d'identifier de manière fiable les plasmocytes régulateurs naturels qui deviennent producteurs d'IL-10 après une infection. Toutefois, des plasmocytes exprimant LAG-3 et produisant de I'IL-10 de manière constitutive sont naturellement présents dans la moelle osseuse de souris naïves. Les plasmocytes $\mathrm{LAG}-3^{+}$produisent en grande majorité des immunoglobulines d'isotype $M(\operatorname{Ig} M)$, ce qui indique qu'ils n'ont pas procédé à une commutation de la classe de leur isotype. Ils expriment aussi, à un niveau plus élevé que les plasmocytes LAG-3-, le facteur de transcription BLIMP-1 (B-lymphocyteinduced maturation protein 1 ) qui est essentiel pour la différenciation des cellules $B$ en plasmocytes et est impliqué dans l'expression du gène codant I'IL-10. L'expression différentielle des gènes peut être expliquée par des particularités épigénétiques. Un processus épigénétique clé est l'ajout d'un groupement méthyle au niveau des sites $C p G$ (cytosine phosphate guanine) de I'ADN génomique par les ADN méthyltransfé- 

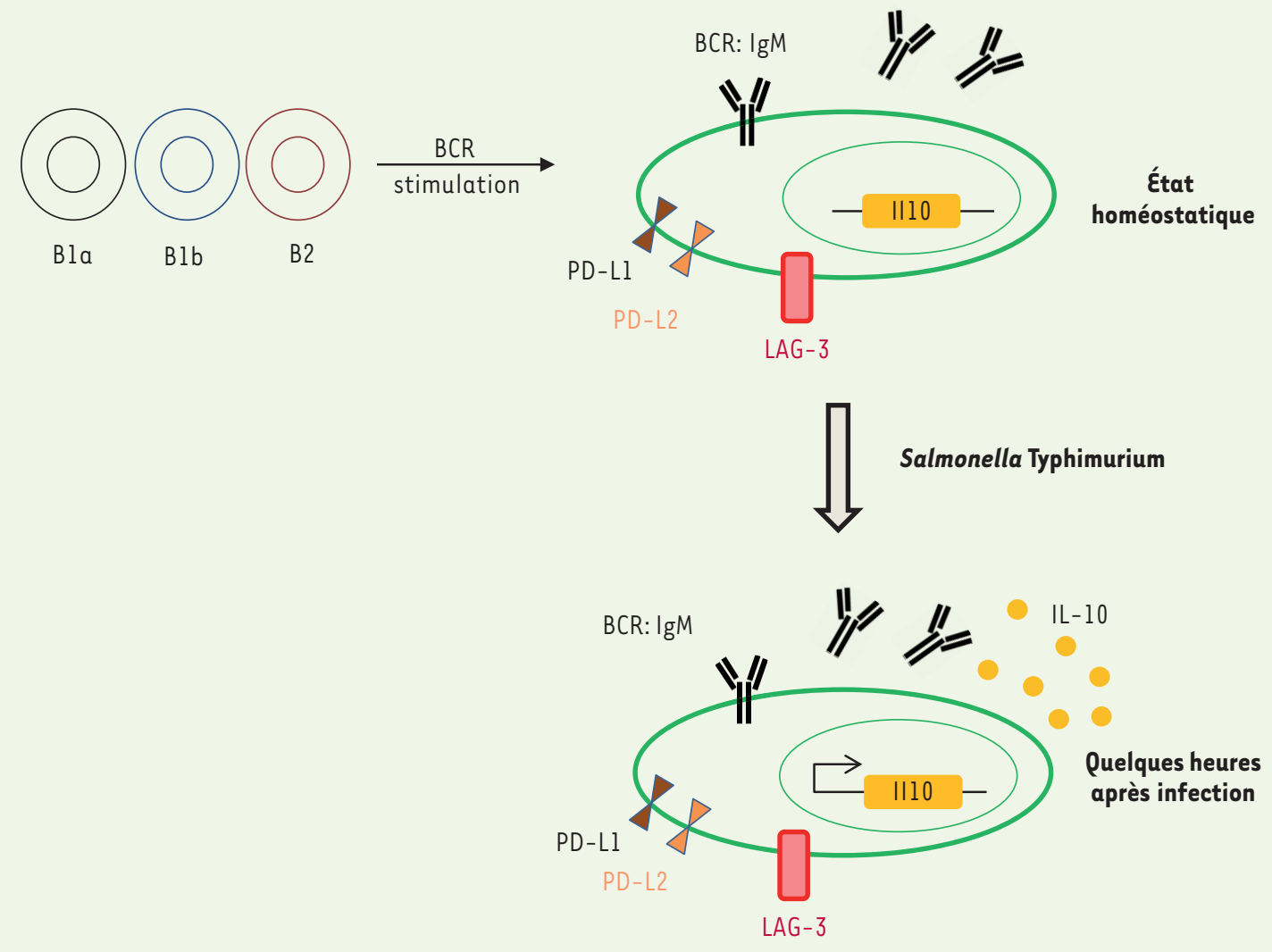

Figure 1. Schéma du développement et de l'activation des plasmocytes naturels CD19+CD138+LAG-3+ chez la souris. À l'état homéostatique, certaines cellules lymphocytaires Bl (Bla et Blb) ainsi que B2 peuvent se différencier via un processus dépendant du BCR (récepteur des cellules B) en plasmocytes spécialisés pour la production de l'IL-10 (interleukine-10). Ces plasmocytes sécrètent des immunoglobulines d'isotype M (IgM) tout en maintenant l'expression de cet anticorps à la surface et se distinguent par l'expression des molécules inhibitrices LAG-3 (lymphocyte activation gene-3), PD-L1, et PD-L2 (progammed death-ligand 1 and 2). Ces cellules ont la propriété caractéristique de produire de l'IL-10 en quelques heures après une infection par la bactérie Salmonella Typhimurium.

rases. Les plasmocytes sont des cellules dont l'ADN est globalement hypométhylé par rapport aux lymphocytes B naïfs. Plus spécifiquement, les plasmocytes régulateurs $\mathrm{LAG}-3^{+}$ont un épigénome distinct de celui des plasmocytes qui n'expriment pas LAG-3, caractérisé par une chromatine moins méthylée et donc en conformation plus ouverte facilitant la transcription au niveau du locus du gène $\| 10$, ce qui pourrait expliquer en partie leur propriété fonctionnelle distincte.

Les signaux induisant la différenciation de ces cellules sont encore mal connus, mais il apparaît que l'émergence des plasmocytes $\mathrm{LAG}-3^{+}$est contrôlée par les voies de signalisation du BCR ( $B$ cell receptor). En effet, ces plasmocytes sont quasiment absents chez les souris déficientes pour Btk (Bruton tyrosine kinase), qui code une enzyme essentielle pour la signalisation du BCR. De même, la proportion de plasmocytes $L A G-3^{+}$est drastiquement diminuée chez les souris n'exprimant pas CD19, un corécepteur activateur du BCR. En revanche, le récepteur CD40 ou les TLR (toll-like receptor) ne sont pas indispensables à leur formation. Comme il a été décrit que la réponse T-dépendante nécessite $C D 40$ et que la réponse T-indépendante de type $l^{1}$ nécessite

\footnotetext{
1 Les réponses de type 1 et 2 diffèrent par le mode d'activation des lymphocytes $B$ impliqués. La réponse de type 1 implique une voie commune d'activation parmi plusieurs lymphocytes B différents, la réponse n'est donc pas spécifique et l'activation est polyclonale.
}

l'engagement des TLR, ces observations suggèrent que les plasmocytes régulateurs naturels $\mathrm{LAG}_{-} 3^{+}$émergent dans le contexte d'une réponse T-indépendante de type $2^{2}$. Les antigènes impliqués dans cette réponse n'ont pas été formellement identifiés, mais ils pourraient provenir de molécules du soi modifiées, car la présence d'un microbiote n'est pas nécessaire à leur émergence. En effet, ils sont présents en quantité normale chez des souris élevées dans un environnement totalement stérile (germ-free).

\footnotetext{
La réponse de type 2, quant à elle, provoque une stimulation monoclonale et spécifique.
} 
L'origine de ces plasmocytes LAG $-3^{+}$a également été étudiée par la détermination du répertoire de leur BCR. Les plasmocytes $L A G-3^{+}$se distinguent de ceux n'exprimant pas LAG-3 par une présence en nombre plus faible de mutations somatiques au niveau des chaînes lourdes d'immunoglobulines. Par ailleurs, une fraction des plasmocytes $L A G-3^{+}$exprime un BCR connu pour reconnaître la phosphatidylsérine, un antigène exposé à la surface des cellules entrant en apoptose. Dans la moelle osseuse de souris naïves, environ un quart des plasmocytes LAG$3^{+} \mathrm{IL}-10^{+}$exprime ce $B C R$, suggérant que les cellules apoptotiques jouent un rôle important dans le développement de ces cellules. De manière plus générale, ceci suggère que les plasmocytes $\mathrm{LAG}-3^{+}$sont induits par des antigènes T-indépendants de type 2 associés à des cellules ou globules rouges endommagés. Ce BCR donne aussi une indication sur les sous-populations de cellules $B$ précurseurs de ces plasmocytes puisqu'il est connu pour être caractéristique des lymphocytes $\mathrm{Bla}^{3}$. Néanmoins, les lymphocytes Blb et B2 sont aussi capables de se différencier en plasmocytes $L A G-3^{+}$. Les plasmocytes

3 Les cellules Bla sont caractérisées par leurs phéno types $C D 19^{+} C D 11 b^{+} C D 5^{+}$dans la cavité péritonéale, et $\mathrm{CD} 19^{+} \mathrm{B} 220^{\text {faible }}$ dans la rate et la moelle osseuse.
$\mathrm{LAG}-3^{+}$ont donc des origines diverses, mais présentent un répertoire $B C R$ qui leur est propre.

À l'état homéostatique, certains lymphocytes B se différencient en plasmocytes ayant un potentiel régulateur. Ceci suggère que ce processus pourrait également se produire au cours de réponses immunitaires. De nombreuses indications et notamment des expériences de transferts adoptifs de lymphocytes B confirment l'hypothèse selon laquelle une différenciation de lymphocytes $B$ en plasmocytes régulateurs induits se produit au cours des réponses immunitaires [6]. Les signaux générant des plasmocytes régulateurs naturels restent à identifier car des immunisations classiques par des antigènes $T$-dépendants ou T-indépendants de type 1 ou 2 n'induisent pas de telles cellules [4].

Enfin, une question importante est celle de l'existence de plasmocytes régulateurs chez l'homme. Plusieurs études ont documenté l'observation de plasmocytes producteurs d'IL-10 ou de la chaîne inductible de l'IL-35 chez I'homme $[8,9]$, mais le rapport entre les plasmocytes murins et leurs équivalents humains reste à définir. $\diamond$ Identification of natural regulatory plasma cells and their role in physiopathology

\section{REMERCIEMENTS}

Nous remercions tous les auteurs et collaborateurs ayant participé à cette étude. Les travaux décrits dans cette nouvelle ont été financés par ERC PREGLAB 647696, AXA Chair Translational Immunology, Chair of Excellence (Université Sorbonne Paris Cité), Infect-ERA project ABIR (031A403), and Deutsche Forschungsgemeinschaft (TRR130, FI 1238/1-2).

\section{LIENS D'INTÉRÊT}

Les auteurs déclarent n'avoir aucun lien d'intérêt concernant les données publiées dans cet article.

\section{RÉFÉRENCES}

1. Shen P, Fillatreau S. Antibody-independent functions of B cells : a focus on cytokines. Nat Rev Immunol $2015 ; 15$ : 441-51.

2. Kaplon K, Dieu-Nosjean MC. Quel avenir pour les lymphocytes B infiltrant les tumeurs solides. Med Sci (Paris) $2018 ; 34: 72-8$.

3. Bouaziz JD, de Masson A, Le Buanec $\mathrm{H}$, et al. Lymphocytes $B$ régulateurs : état des connaissances. Med Sci (Paris) 2014 ; 30 : 721-4.

4. Lino AC, Dang VD, Lampropoulou V, et al. LAG-3 inhibitory receptor expression identifies immunosuppressive natural regulatory plasma cells. Immunity 2018; 49: 120-33 e9.

5. Wang J, Sanmamed MF, Datar I, et al. Fibrinogen-like protein 1 Is a major immune inhibitory ligand of LAG3. Cell 2019 ; 176 : 334-47 el2.

6. Fillatreau S. Natural regulatory plasma cells. Curr Opin Immunol 2018 ; $55: 62-6$.

7. Champiat S, Soria JC. Nouvelles pratiques en immunooncologie: une révolution et un immense défi scientifique et médical. Med Sci (Paris) 2017; 33:563-4.

8. Machado-Santos J, Saji $\varepsilon$, Troscher AR, et al. The compartmentalized inflammatory response in the multiple sclerosis brain is composed of tissueresident $C D 8^{+} T$ lymphocytes and B cells. Brain 2018 ; $141: 2066-82$.

9. Larousserie F, Bardel $\varepsilon$, Coulomb L'Hermine A, et al. Variable expression of Epstein-Barr virus-induced gene 3 during normal $B$-cell differentiation and among B-cell lymphomas. J Pathol 2006 ; 209 : 360-8.

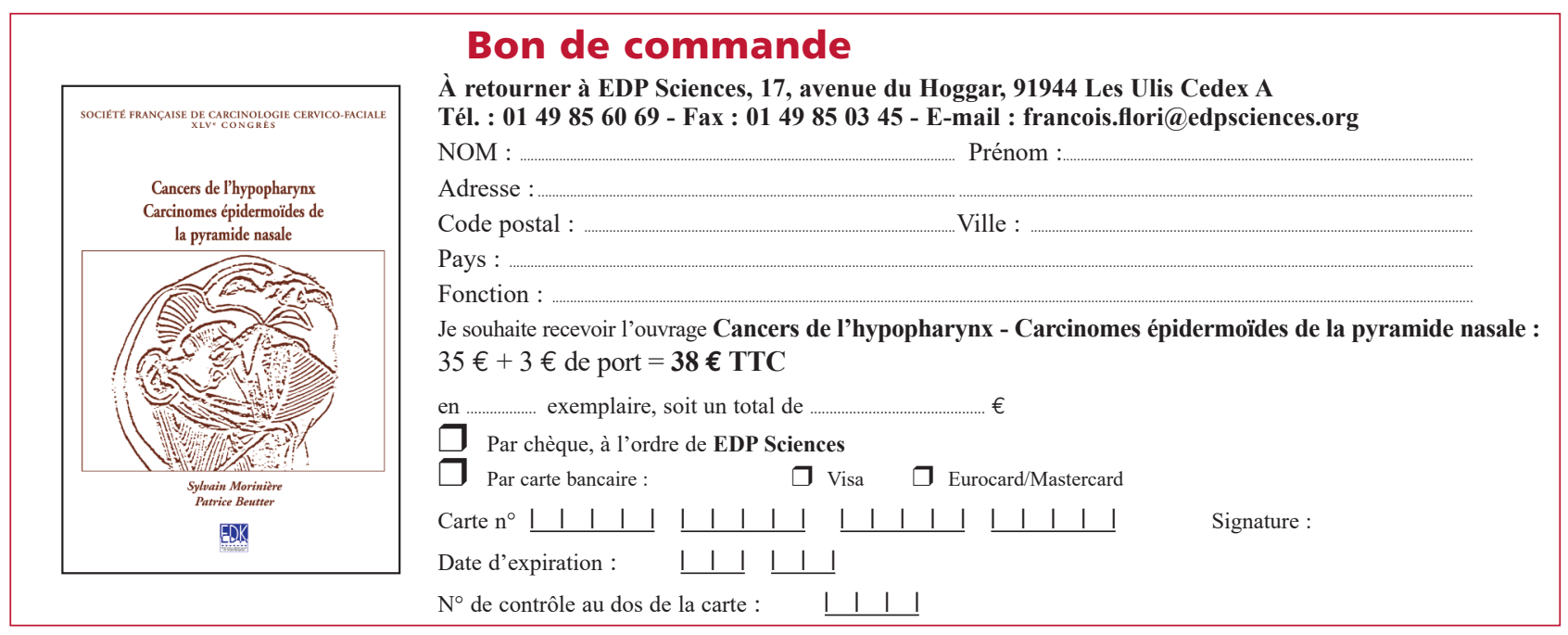

